\title{
Molecular characterization and diagnostic investigations of rabies encephalitis in camels (Camelus dromedaries) in Oman: a retrospective study
}

\author{
Mohamed S. Ahmed ${ }^{1,2} \cdot$ Mohammad H. Body $^{1} \cdot$ Mahmoud S. El-Neweshy ${ }^{3,4}\left(\mathbb{D} \cdot\right.$ Abdulmajeed H. ALrawahi $^{1}$. \\ Mohsin Al-Abdawani ${ }^{1} \cdot$ Hatim A. Eltahir ${ }^{1,5}$ - Mahir G. ALmaewaly ${ }^{1}$
}

Received: 11 December 2019 / Accepted: 13 February 2020 / Published online: 2 March 2020

(C) Springer Nature B.V. 2020

\begin{abstract}
The accurate and early diagnosis of rabies is critical for undertaking public health measures in animals. The aim of the current study was to identify the molecular characterization of the circulating rabies virus (RABV) among camels (Camelus dromedaries) in Oman and to evaluate the efficacy of the histopathology and reverse transcription polymerase chain reaction (RT-PCR) as diagnostic tools of acute rabies encephalitis in camels in comparison with direct fluorescent antibody test (dFAT). Of the fortyfive brain samples from suspected camels submitted to the Animal Health Research Center in Oman (2009-2013), 22 cases were positive by dFAT and RT-PCR. Two positive samples were subjected for $\mathrm{N}$ gene nucleotide sequencing and phylogenetic analysis (accession numbers GU353156 and KC883998 for brain samples collected in 2009 and 2011, respectively). The specificity and sensitivity of histopathology were $100 \%$ and $81 \%$, respectively, while in RT-PCR were $100 \%$ and $100 \%$, respectively. The neuropathological changes were presence of intracytoplasmic inclusions (Negri bodies) in the pyramidal neurons of the hippocampus beside prominent cerebral and cerebellar congestion and hemorrhage. Neuronal necrosis with satellitosis and neuronophagia were also noticed in the cerebrum of affected brains. Conclusively, there was one genetic group of RABV with 99\% homology circulating in Omani camels. Also, it is concluded that histopathological examination is a safe and reliable diagnostic tool when only formalin-fixed and paraffin-embedded material is available, but the negative results should be reaffirmed by dFAT or RT-PCR.
\end{abstract}

Keywords Rabies $\cdot$ Camel $\cdot$ Molecular $\cdot$ Diagnosis $\cdot$ Histopathology $\cdot$ Oman

Mahmoud S. El-Neweshy

mahmoud.neweshy@alexu.edu.eg

1 Animal Health Research Center, Directorate General of Agriculture and Livestock Research, Ministry of Agriculture and Fisheries, Muscat, Sultanate of Oman

2 Department of Pathology, Faculty of Veterinary Medicine, Kafrelsheikh University, Kafr El Sheikh 33516, Egypt

3 Department of Pathology, Faculty of Veterinary Medicine, Alexandria University, Edfina, Behera, Alexandria 22785, Egypt

4 Central Laboratory for Animal Health, Ministry of Agriculture and Fisheries, Muscat, Sultanate of Oman

5 Central Veterinary Research Laboratory, Khartoum, Sudan

\section{Introduction}

Rabies is an infectious, zoonotic viral disease that affects the central nervous system of all warm-blooded animals including humans resulting in severe encephalomyelitis (Hanlon and Childs 2013).Rabies caused by rabies virus (RABV), a negative-stranded RNA virus, belong to the genus Lyssavirus of Rhabdoviridae family. Rabies is a continuous public health hazard that causes about 59,000 human deaths annually worldwide, most of them being in Asia and Africa, particularly in developing countries (Hampson et al. 2015; Parviz et al. 2004).

There are two types of rabies either urban or sylvatic. The major reservoir hosts in urban type are dogs and cats, while the major reservoir hosts in sylvatic type are foxes, wolves, bats, and other wildlife (WHO 2013).

Livestock species usually get rabies through bites of rabid dogs and infrequently through bites of rabid carnivores. However, other routes such as aerosol, ingestion, and 
transplantation have also been documented (Body et al. 2014; Sudarshan 2005).

Severe agitation, depression, hydrophobia, and paralysis followed by impaired consciousness and coma are the constant clinical signs of rabies (WHO 2013). Eosinophilic intracytoplasmic inclusions known as Negri bodies are the pathognomonic histopathological lesions of rabies (Duong et al. 2016; Woldehiwet 2005). The occurrence of Negri bodies was found to be related to the rabid animal species, pyramidal cells within Ammon's horn of the hippocampus in dogs, and Purkinje cells of the cerebellum in cow and buffalo (Jamadagni et al. 2007). Rapid dissemination of RABV in CNS associated with prominent neuropathological changes (perivascular lymphocytic cuffing, satellitosis, neuronal necrosis, and neuronophagia) indicates rapid progressive encephalitis (Fishbein and Bernard 2014).

Clinical diagnosis of rabies in live animals is difficult and usually performed during necropsy, so early diagnosis of rabies in animals is critical for postexposure prophylaxis administration (Zimmer et al. 1990). Many laboratory techniques are used in rabies diagnosis such as direct fluorescent antibody test (dFAT) which consider as the gold-standard diagnostic technique, reverse transcription polymerase chain reaction (RT-PCR) (Manjunathareddy et al. 2016), and histopathological examination of the brain for Negri bodies. All tests require fresh tissue samples that represent great hazard of possible risk of contamination of the environment with rabies virus (Abreu et al. 2012).

So, the aim of the current study is to identify the molecular characterization of the circulating rabies virus among camels in Oman and to evaluate the diagnostic efficacy of histopathology and RT-PCR for rabies encephalitis in camels in comparison with dFAT.

\section{Materials and methods}

Whole brains were collected during a 5-year period (20092013) from 45 apparently rabid camels exhibiting one, some, or all of the following signs: excitation, foamy saliva at mouth, yawning, self-biting, pica, ataxia, altered phonation, restlessness, rotation of head and neck in all directions, sternal recumbency, and paresis besides a fresh or cicatricle bite wound. The examined brains showed severe congestion and slight edema of meninges, cerebellum, and cerebral hemisphere besides hemorrhage. Most brains showed cerebral broad gyri and narrow sulci. Three brain samples (each sample involved parts from cerebrum, cerebellum, and medulla oblongata) were prepared for dFAT, RT-PCR, and histopathology.

Regarding dFAT, brain impression smears were made, fixed with acetone, then were stained with fluorescein isothiocyanate (FITC)-anti-rabies monoclonal globulin conjugate (Fujirebio Diagnostics Inc.) for $30 \mathrm{~min}$ at $37^{\circ} \dot{\mathrm{C}}$ in humid chamber as described previously (Wiktor et al. 1980). The slides were washed three times with PBS on magnetic stirrer in order to create turbulence over. Thereafter, air dried, mounted in $90 \%$ glycerin buffer $(\mathrm{pH} 8.5)$ and covered with a cover slip, and examined under the fluorescent microscopy.

Concerning RT-PCR, RNA extraction was carried out from $10 \mathrm{mg}$ of brain samples using QIAamp viral RNA kit (Qiagen). Using Qiagen one-step RT-PCR kit, 100 ng RNA was adopted as template for cDNA (Singh et al. 2010). The PCR amplification of $355 \mathrm{pb}$ were analyzed on $1.5 \%$ agarose gel containing $0.7 \%$ ethidium bromide.

$\mathrm{N}$ gene nucleotide were sequenced using the ABI PRISM BigDye terminator cycle sequencing kit (Perkin Elmer, USA), and the phylogenetic tree was constructed using neighborjoining method (Saitou and Nei 1987).

For histopathology, brain specimens were directly fixed in $10 \%$ neutral buffered formalin. Thereafter, the fixed specimens were processed for paraffin-embedded sections, then stained with hematoxylin and eosin (H\&E) (Bancroft and Gamble 2008).

\section{Results}

\section{dFAT}

Among 45 rabies-suspected camels, 22 were found positive for rabies virus by dFAT and RT-PCR. Like the positive control slide, positive samples showed bright green fluorescent foci of varying size from dust particles to prominent inclusion scattered within the smear (Fig. 1). While in negative control slide, no antigen expression was noticed.

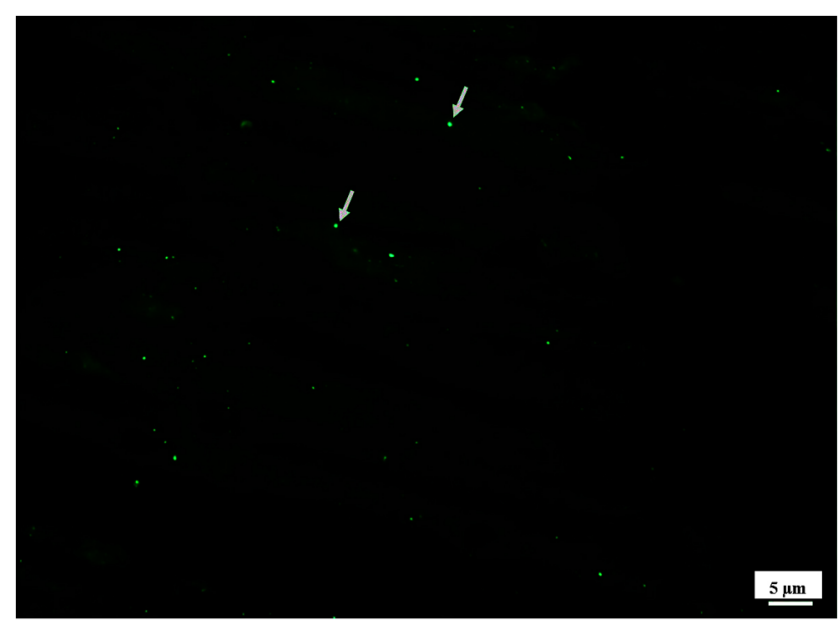

Fig. 1 Brain impression of 3-year-old rabid camel tested by direct fluorescent antibody test (DFT) showing rabies virus antigen which appear as small (dust like) immunofluorescent apple-green particles (arrows) 


\section{RT-PCR}

The expected size product of $320 \mathrm{bp}$ was got amplified in all dFAT-positive samples only from rabies-suspected camels.

\section{Sequencing of $\mathbf{N}$ gene nucleotide}

Two positive samples collected in 2009 and 2011 were sequenced and submitted to the GenBank with accession number GU353156 and KC883998 (Body et al. 2014), respectively. The molecular and phylogenetic analysis of the nucleotide sequence of the $\mathrm{N}$ gene showed that there is one genetic group of RABV with $99 \%$ homology (Fig. 2).

\section{Histopathology}

All dFAT-positive samples (22 samples) showed perivascular lymphocytic and monocytic cuffs indicative of nonsuppurative viral encephalitis and congestion. The cerebrum exhibited the presence of Negri bodies in the hippocampal pyramidal neurons (Fig. 3a) in $81.8 \%$ dFAT-positive samples. Only 20 brains of dFAT-positive samples (90.9\%) showed neuronal necrosis (Fig. 3b), neuronophagia babe's nodules (Fig. 3c), and neuronal degeneration and necrosis.
Compared to dFAT- positive samples, most samples (86.36\%) showed neuronal cell swelling, cytoplasmic vacuolation, and gliosis. Also, less prevalent histopathological changes were noticed including marked edema and diffuse hemorrhages (Fig. 3d) in $72.7 \%$, satellitosis and meningitis in $31.8 \%$, and malacia in $36.36 \%$. All dFAT- negative brain samples were histologically normal.

\section{Discussion}

Camels in Oman are one-humped dromedary camel (Camelus dromedaries), which constitute an important proportion of livestock with a population number approximately 130,000 heads (MAF 2013). Rabies is one of the most important zoonotic diseases causing progressive encephalitis. Sylvatic rabies is endemic in Oman (Al Ismaily et al. 2002; Body et al. 2014; Hussain et al. 2013) where bite of rabid red foxes (Vulpes vulpes) is central of sylvatic rabies transmission (Scrimgeour and Mehta 2001) and associated with human and animal cases since the first outbreak in 1990 (Al Ismaily et al. 2002; Scrimgeour and Mehta 2001). In the current study, most rabid camels were bitten by foxes which confirm the most frequent natural route of spreading of RABV in Oman,
Fig. 2 Phylogenetic tree of N gene nucleotide sequences of two $\mathrm{RABV}$ isolates from Omani camels using neighbor- joining method

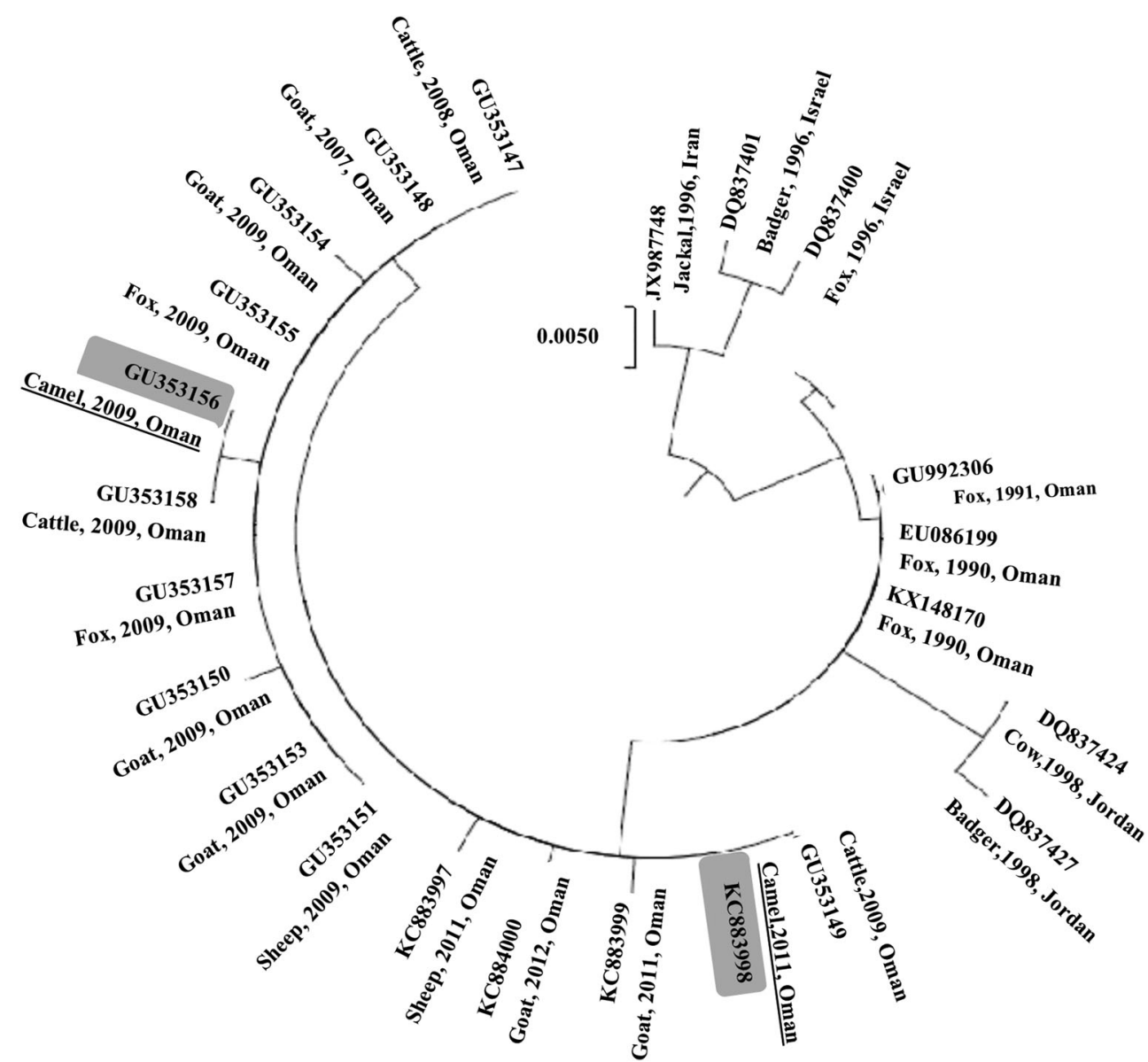




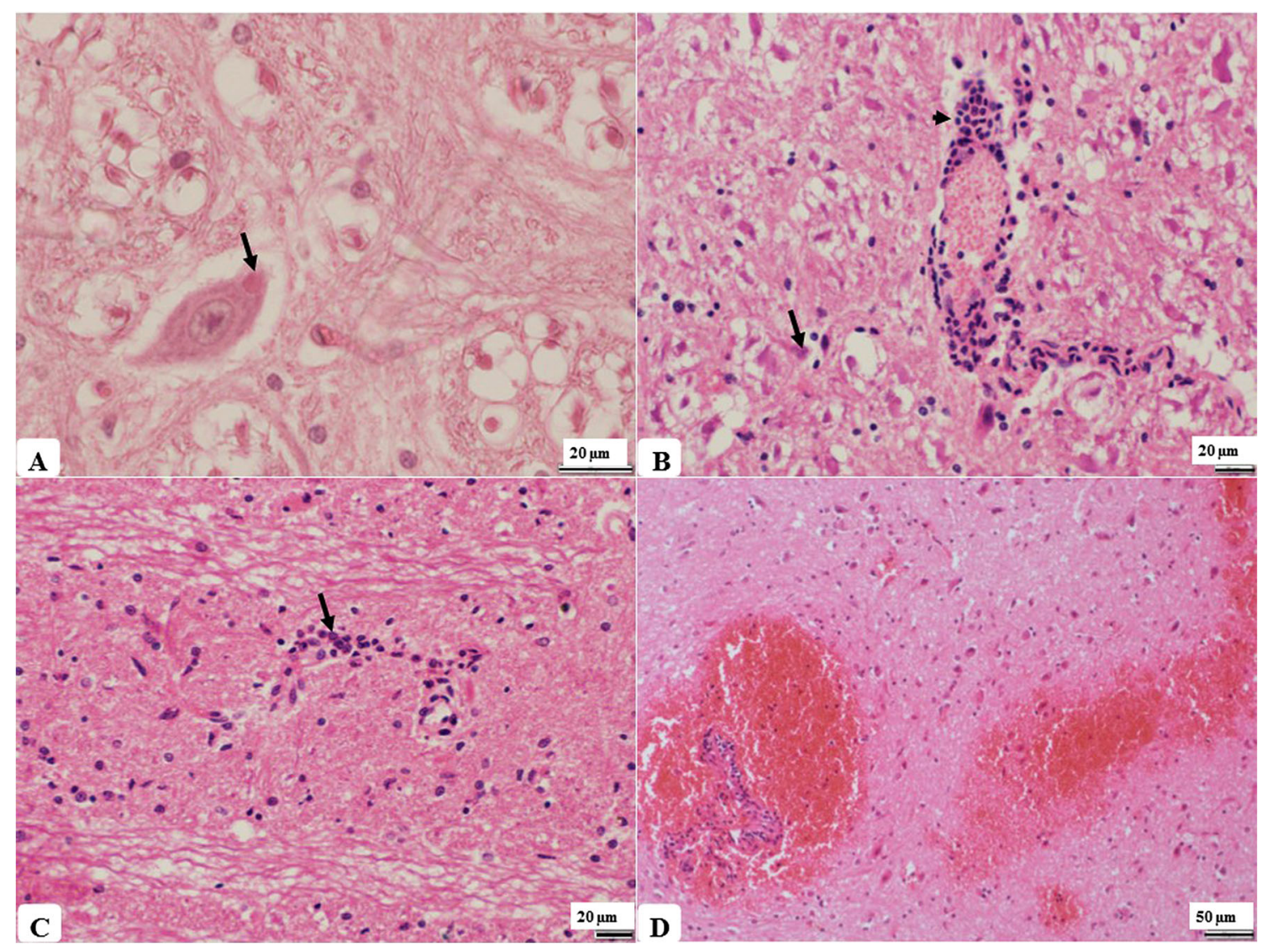

Fig. 3 Histopathology of rabid camel's brain showing. a Intracytoplasmic oval eosinophilic body (Negri body; arrow). b

(long arrow). c Focal collection of microglial cell (Babe's bodies; arrow). d Massive perivascular and parenchymal hemorrhage

Lymphocytic perivascular cuffing (short arrow) and neuronal necrosis

while the biting by other carnivores like dog might be considered in few cases. Due to their free-roaming nature, camels have a greater chance to come in contact with wild live foxes.

In Oman, all bitten camels were considered rabid and managed with isolation, management of bite wound, postexposure vaccination, close observation, and postmortem laboratory confirmation of clinically rabid animals using brain tissue followed by ring vaccination in confirmed cases.

The molecular epidemiological studies on rabies among animals in Oman since first reported cases in 1990 based mainly on $\mathrm{N}$ gene sequences of RABV (Al Abaidani et al. 2015; Body et al. 2018; Body et al. 2014; Hussain et al. 2013), so the current study used $\mathrm{N}$ gene sequences to be related with the others local studies. The phylogenetic analysis revealed that one genetic group with $99 \%$ homology RABV is circulating among the camels in Oman during the period of study. Body et al. (2014) demonstrated a high similarity between RABV isolates from different animal species in different geographical regions in Oman.

Clinical diagnosis of rabies is not easy and confused with others neurotropic virus-induced encephalitis or with normal aggressive tendencies of the animals and in those suddenly developed obvious behavioral changes especially in unvaccinated animals (Jamadagni et al. 2007).

Few pathological lesions such as cerebral edema were noticed although the dramatic and severe clinical course of rabid animals (Murphy 1977) which may be attributed to minimal vascular changes and subtle lesions in the lymphocytic inflammation.

In the current study, Negri bodies were found in $81.8 \%$ of dFAT and RT-PCR. Toll-like receptor 3 (TLR3), a cell protein, signals the virally infected cell to produce interferons. When formed, it is relocated to Negri body, so it is required for its formation. In RABV-infected cell, TLR3 level is reduced by RNA silencing. Negri bodies not formed at this early point. Later in clinical disease, rabies virus RNA and viral proteins aggregate in the cytoplasm forming Negri bodies (Menager et al. 2009).Singh and Grewal (1998) recorded that Negri bodies was considered the defining diagnostic test for positive identification of a rabies infection, and only about $50-80 \%$ of rabies positive animals actually have Negri bodies. Other reports (Jamadagni et al. 2007; Singh 2001) stated that the presence of Negri bodies is a unique and pathognomonic finding in rabies infection and indicates rabies disease. 
In the present study, inflammatory reactions with the presence of perivascular aggregation of lymphocytes and monocytes, babe's nodules, satellitosis, and meningitis were prominent in the cerebrum of affected camels which agreed with Jamadagni et al. (2007). Neuronal loss such as neuronal necrosis, neuronophagia, necrotic degeneration in neuronal cells, and malacia in the cerebrum were observed in majority of samples as mentioned by Li et al. (2005).

Bright green fluorescent particles were observed in the brain tissue smears by dFAT in $53.6 \%$ of suspected samples which were diagnosed positive. The WHO currently reports dFAT to be as the gold standard for detection of rabies diagnosis (Hayman et al. 2011). dFAT improved diagnostic accuracy by promoting visualization of RABV and enabled detection of antigens immunogenically altered by fixation (Rissi et al. 2008).

In the present study, the sensitivity (true positive rate) and specificity (true negative rate) of RT-PCR were $100 \%$ and $100 \%$, respectively compared to dFAT results. David et al. (2002) reported the high accuracy of RT-PCR in detection of RABV in decomposed brain samples that are negative by dFAT.

Rabies is usually diagnosed at necropsy by dFAT or histopathology using fresh or formalin-fixed brain samples, respectively. Although dFAT is the standard technique for rabies diagnosis, use of fresh brain samples in this technique may become hazardous with possible risk of environmental contamination with RABV which completely inactivated by the fixative "acetone" (Abreu et al. 2012; Jarvis et al. 2016; Woldehiwet 2005). Also the unsuitability of highly decomposed samples and need for well-equipped laboratories limit the wide usage of FAT for rabies diagnosis (Dürr et al. 2008). In spite of the many advantages of dFAT in diagnosis of rabies but warm climate and mishandling during transportation of fresh brain samples in countries where diagnostic laboratories are not well established could interfere with the result (Prabhu et al. 2014; Stein et al. 2010). Meanwhile, fixation of brain tissues in formalin for rabies diagnosis simplifies collection, storage, and transportation of samples simple and eliminates hazards.

It can be concluded that one genetic RABV group with 99\% homology circulates in Omani camels, with no evidence of any other variant existed in Omani camels. Also the histopathological diagnosis of suspected rabid camels is a safe and reliable laboratory tool when only formalin-fixed and paraffin-embedded material is available, but the negative results should be confirmed by dFAT or RT-PCR.

The controlling of sylvatic rabies should be the main goal of any control program in Oman. The prophylactic rabies vaccination could be recommended in the camel population living near the areas favoring the fox habitat (mountainous terrain) that will decrease the incidence of rabies in camels of Oman.
Funding information This study was co-funded by the Agriculture and Fisheries Development Fund (AFDF), Ministry of Agriculture and Fisheries, Sultanate of Oman. AFDF had no role in data collection, analysis, or decision to publish.

\section{Compliance with ethical standards}

Conflict of interest The authors declare that they have no conflict of interest.

\section{References}

Abreu, C.C. et al., 2012. Domestic microwave processing for rapid immunohistochemical diagnosis of bovine rabies, Histology and histopathology, 12, 1227-1230

Al Abaidani, I. et al., 2015. Epidemiology of rabies in Oman: a retrospective study [1991-2013], EMHJ-Eastern Mediterranean Health Journal, 21, 591-597

Al Ismaily, S. et al., 2002. Retrospective studies of rabies in the Sultanate of Oman 1990-2000, Agric. Fish. Res. Bull, 2, 25-28

Bancroft, J.D., and Gamble, M., 2008. Theory and practice of histological techniques, (Elsevier health sciences)

Body, M.H.H. et al., 2014. Study on molecular characterization of rabies virus $\mathrm{N}$ gene segment from different animal species in the Sultanate of Oman, Journal of Veterinary Medicine and Animal Health, 6, 295-301

Body, M.H. et al., 2018. Molecular Characterization of Rabies Virus from Foxes in the Sultanate of Oman, Animal and Veterinary Sciences, 6, 17

David, D. et al., 2002. Rabies virus detection by RT-PCR in decomposed naturally infected brains, Veterinary microbiology, 87, 111-118

Duong, V. et al., 2016. Laboratory diagnostics in dog-mediated rabies: an overview of performance and a proposed strategy for various settings, International journal of infectious diseases, 46, 107-114

Dürr, S. et al., 2008. Rabies diagnosis for developing countries, PLoS neglected tropical diseases, 2, e206

Fishbein, D., and Bernard, K., 2014. Rabies virus. In: D.u.a.B. Mandell (ed), Principle and practice of infectious diseases, NewYork Churchill Livingstone, 1527-1541.

Hampson, K. et al., 2015. Estimating the global burden of endemic canine rabies, PLoS neglected tropical diseases, 9, 1-20

Hanlon, C., and Childs, J., 2013. Rabies: Scientific Basis of the disease and its management, Rabies Epidemiology, second editions edn, 61-107

Hayman, D.T. et al., 2011. A universal real-time assay for the detection of Lyssaviruses, Journal of Virological Methods, 177, 87-93

Hussain, M.H. et al., 2013. Spatio-temporal pattern of sylvatic rabies in the Sultanate of Oman, 2006-2010, Preventive veterinary medicine, 110, 281-289

Jamadagni, S., Singh, C., and Sandhu, B., 2007. Histopathological alterations in brains of rabies infected buffaloes and cattle, Italian Journal of Animal Science, 6, 872-874

Jarvis, J.A., Franke, M.A., and Davis, A.D., 2016. Rabies direct fluorescent antibody test does not inactivate rabies or eastern equine encephalitis viruses, Journal of Virological Methods, 234, 52-53

Li, X.-Q., Sarmento, L., and Fu, Z.F., 2005. Degeneration of neuronal processes after infection with pathogenic, but not attenuated, rabies viruses, Journal of virology, 79, 10063-10068

MAF, 2013. Agricultural Statistics Database. https://www.oman.om/wps/ portal/index/gov/omanStatistics/agriculturalCensus/

Manjunathareddy, G. et al., 2016. Diagnosis of animal rabies: comparison of direct fluorescence test (dFAT), reverse transcriptase pcr and realtime PCR 
Menager, P. et al., 2009. Toll-like receptor 3 (TLR3) plays a major role in the formation of rabies virus Negri Bodies, PLoS pathogens, 5, e1000315

Murphy, F., 1977. Rabies pathogenesis, Archives of virology, 54, 279297

Parviz, S. et al., 2004. Rabies deaths in Pakistan: results of ineffective post-exposure treatment, International journal of infectious diseases, $8,346-352$

Prabhu, K. et al., 2014. Application of N-protein monoclonal antibody based direct fluorescent antibody assay (DFA) and direct rapid immunohistochemistry test (dRIT) for detection of rabies virus in brain samples of animals in India, Commonwealth Veterinary Journal, 30, $11-16$

Rissi, D.R. et al., 2008. Occurrence of rabies in sheep in Rio Grande do Sul, Brazil, Pesquisa Veterinária Brasileira, 28, 495-500

Saitou, N., and Nei, M., 1987. The neighbor-joining method: a new method for reconstructing phylogenetic trees, Molecular biology and evolution, 4, 406-425

Scrimgeour, E.M., and Mehta, F.R., 2001. Rabies in Oman: Failed postexposure vaccination in a lactating woman bitten by a fox, International journal of infectious diseases, 5, 160-162

Singh, C., 2001. Laboratory Diagnosis Of Rabies By Elisa In Buffalo Calves Experimentally Infected With Rabies Virus, (Guru Angad Dev Veterinary and Animal Sciences University, Ludhiana)

Singh, C., and Grewal, G., 1998. Early pathogenic study in experimental rabies in buffalo calves with street rabies virus, Buff. J, 3, 361-373
Singh, K. et al., 2010. Detection of rabies virus in brain tissue by one-step reverse transcription-polymerase chain reaction, Indian Journal of Veterinary Pathology, 34, 1-4

Stein, L. et al., 2010. Immunohistochemical study of rabies virus within the central nervous system of domestic and wildlife species, Veterinary Pathology, 47, 630-633

Sudarshan, M., 2005. Assessing burden of rabies in India: WHO sponsored national multicentric rabies survey, 2003, Indian Journal of Community Medicine, 30, 100

WHO, 2013. WHO expert consultation on rabies: second report, (World Health Organization)

Wiktor, T., Flamand, A., and Koprowski, H., 1980. Use of monoclonal antibodies in diagnosis of rabies virus infection and differentiation of rabies and rabies-related viruses, Journal of Virological Methods, $1,33-46$

Woldehiwet, Z., 2005. Clinical laboratory advances in the detection of rabies virus, Clinica Chimica Acta, 351, 49-63

Zimmer, K. et al., 1990. Evaluation of five different methods for routine diagnosis of rabies, Journal of Veterinary Medicine, Series B, 37, $392-400$

Publisher's note Springer Nature remains neutral with regard to jurisdictional claims in published maps and institutional affiliations. 